\title{
SOME THOUGHTS ON THE COSMOLOGICAL QCD PHASE TRANSITION*
}

\author{
W-Y. P. HWANG \\ The Leung Research Center for Cosmology and Particle Astrophysics, \\ Center for Theoretical Sciences, Institute of Astrophysics, \\ and Department of Physics, National Taiwan University, Taipei 106, Taiwan \\ wyhwang@phys.ntu.edu.tw
}

\begin{abstract}
The cosmological QCD phase transitions may have taken place between $10^{-5} \mathrm{~s}$ and $10^{-4} \mathrm{~s}$ in the early universe offers us one of the most intriguing and fascinating questions in cosmology. In bag models, the phase transition is described by the first-order phase transition and the role played by the latent "heat" or energy released in the transition is highly nontrivial and is being classified as the first-order phase transition. In this presentation, we assume, first of all, that the cosmological QCD phase transition, which happened at a time between $10^{-5} \mathrm{~s}$ and $10^{-4} \mathrm{~s}$ or at the temperature of about $150 \mathrm{MeV}$ and accounts for confinement of quarks and gluons to within hadrons, would be of firstorder. Of course, we may assume that the cosmological QCD phase transition may not be of the first-order. To get the essence out of the first-order scenario, it is sufficient to approximate the true QCD vacuum as one of possibly degenerate vacua and when necessary we try to model it effectively via a complex scalar field with spontaneous symmetry breaking. On the other hand, we may use a real scalar field in describing the non-first-order QCD phase transition. In the first-order QCD phase transition, we could examine how and when "pasted" or "patched" domain walls are formed, how long such walls evolve in the long run, and we believe that the significant portion of dark matter could be accounted for in terms of such domain-wall structure and its remnants. Of course, the cosmological QCD phase transition happened in the way such that the false vacua associated with baryons and many other color-singlet objects did not disappear (that is, using the bag-model language, there are bags of radius 1.0 fermi for the baryons) - but the amount of the energy remained in the false vacua is negligible by comparison. The latent energy released due to the conversion of the false vacua to the true vacua, in the form of "pasted" or "patched" domain walls in the short run and their numerous evolved objects, should make the concept of the "radiation-dominated" epoch, or of the "matter-dominated" epoch to be reexamined.
\end{abstract}

Keywords: Cosmology; origin and formation of the universe.

* This paper will also appear in the Proceedings of the Conference in Honor of C. N. Yang's 85th Birthday, to be published by World Scientific and NTU. 


\section{Introduction}

I'm sorry that the time limit for me forced me give a lousy talk - so I try to compensate on that. The other excuse is my experience of the cerebral haemorrhage about three and a half years ago. But this occasion of C. N. Yang's 85th Birthday Symposium, I should complain about my own lousiness and try to quote a sentence from C. N. Yang from "Einstein's Impact on Theoretical Physics in the 21st Century": ${ }^{1}$

\section{"... It led to the discipline of modern cosmology which is destined to become one of the important scientific fields of the 21st century."}

I should say to admire C. N. Yang in his saying his prediction - in fact, I started working on this "field" about eight years ago and start feeling maybe the same thing. I think that we, humankind, should be proud of that cosmology is becoming the empirical science in the 21st century.

Indeed, the discovery ${ }^{2-4}$ of fluctuations or anisotropies, at the level of $10^{-5}$, associated with the cosmic microwave background (CMB) has helped transformed the physics of the early universe into a main-stream research area in astronomy and in particle astrophysics, both theoretically and observationally (Ref. 5, for an early general review). CMB anisotropies ${ }^{6}$ and polarizations, ${ }^{7}$ the latter even smaller and at the level of $10^{-7}$, either primary (as imprinted on the last scattering surface just before the universe was $(379 \pm 8) \times 10^{3}$ years old) or secondary (as might be caused by the interactions of CMB photons with large-scale structures along the line of sight), are linked closely to the inhomogeneities produced in the early universe.

On the other hand, over the last three decades, the Standard Model of particle physics has been well established to the precision level of $10^{-5}$ or better in the electroweak sector, or to the level of $10^{-3}-10^{-2}$ for the strong interactions. In the theory, the electroweak (EW) phase transition, which endows masses to the various particles, and the QCD phase transition, which gives rise to confinement of quarks and gluons within hadrons in the true QCD vacuum, are two well-established phenomena. Presumably, the EW and QCD phase transitions would have taken place in the early universe, respectively, at around $10^{-11} \mathrm{~s}$ and at a time between $10^{-5} \mathrm{~s}$ and $10^{-4} \mathrm{~s}$, or at the temperature of about $300 \mathrm{GeV}$ and of about $150 \mathrm{MeV}$, respectively. Indeed, it has become imperative to formulate the EW and QCD phase transitions in the early universe if a quantitative theory of cosmology can ever be reached.

The purpose of this presentation is to focus our attention on cosmological QCD phase transition and to assess whether its roles in the early universe can be synthesized in a more quantitative terms. To simplify the situation, we use the bagmodel language (and thus the first-order QCD phase transition) as the zeroth-order approximation and, whenever necessary, try to model the possibly degenerate vacua, the lower-temperature phase, as the minima of the spontaneously-broken complex scalar fields. Because we use the bag-model language as the approximation, the phase transition in this case is of first order in nature - in other words, the latent "heat" or energy is all released at the critical temperature $T_{c}$. Whether a given 
amount of "latent energy" is released by the system is of importance — in bag models, the answer is definitely "yes" judging from the difference of the vacua. Owing to the high computation costs, determination of the order of QCD phase transition remains to be a great challenge in lattice calculations. To summarize the situations, ${ }^{8,9}$ the order of the transition depends on the quark mass; for example, in three-flavor QCD for vanishing quark masses the transition is of first-order; but for intermediate masses it is probably a crossover; however, for infinitely heavy quark masses the transition is again first-order. In three-flavor unimproved staggered QCD, using a lattice spacing of about $0.28 \mathrm{fm}$, the first-order and the crossover regions are separated by a pseudoscalar mass of $m_{\pi, c} \approx 300 \mathrm{MeV}$. Studying the same three-flavor theory with the same lattice spacing, but with an improved $p 4$ action, they ${ }^{8,9}$ obtained $m_{\pi, c} \approx 70 \mathrm{MeV}$. So, in the first approximation, a pseudoscalar mass of $140 \mathrm{MeV}$ (which corresponds to the numerical value of the physical pion mass) would be first-order; but in the second approximation, it would be crossover.

In lattice calculations, the minimum of the error bars is what you cannot get rid of. The conceptual errors in the "physical" quark masses might eventually enter the game since after all we cannot observe an isolated quark and cannot weigh it. It seems to me that, in some cases, it might be possible to rule out the first-order or second-order phase transition or some higher-order transition; numerically, it might be very hard to determine the crossover "transition." All in all, we are afraid that the question about the first-order QCD phase transition would remain to be controversial for some time to come. In fact, to say that the cosmological QCD phase transition is NOT first-order is equivalent to saying that bag models are wrong - this may be right but we need more in the proof. In this presentation, we use the bag-model approximation to simplify the complications of the problem in the context of cosmology. That means that we assume the existence of the firstorder QCD phase transition — perhaps with too much of latent "heat" but the amount of latent "heat," even by reduction of three or four orders of magnitude, would still make the claim of the present paper valid.

Usually we treat the ground state as being nondegenerate. This might not be the case - owing to the many degrees of freedom associated with QCD such as the sixteen gauge degrees of freedom. This is true when one tries to work out the ground state in a given model, such as trying to obtain the QCD vacuum in the instanton liquid model. ${ }^{\text {a }}$ This aspect is different from the so-called $\theta$ vacuum associated with the strong CP problem ${ }^{11}$ — there is no degeneracy problem there.

Owing to the complexities of our problem, we could try to tackle the problem of the phase transition by dividing it into problems in four different categories, viz.: (1) how a bubble of different vacuum grows or shrinks; (2) how two growing bubbles collide or squeeze, and merging, with each other; (3) how the universe eventually stabilize itself later while keeping expanding for several orders of magnitude; and

${ }^{a}$ Of course, the ground state in the simplest case is nondegenerate but in realistic cases the problem of the degenerate vacua is rather common, see Ref. 10. 
(4) how specific objects, such as back holes or magnetic strings, get produced during the specific phase transition. Although the system "temperature" kept decreasing, the meaning of the "temperature" may have lost meaning in the local sense during each of the substeps - try to consult the textbooks on nonequilibrium statistical mechanics.

One reference that is highly relevant for our paper is by Svetitsky and Yaffe, ${ }^{12}$ where as one integrates out all degrees of freedom except this order parameter and obtains the effective theory for the order parameter, globally invariant under the center symmetry (i.e. a global symmetry group, the center of the SU(3) gauge group). Of course, when for some good reason, we keep two degrees of freedom unintegrated, we would be left out a complex scalar field instead. In what follows, we use a complex scalar field since the real scalar could be handled with relative ease by comparison.

Questions related to part (4) (i.e. that how specific objects, such as black holes or magnetic strings, get produced during the specific phase transition), which are quite complicated, will not be addressed here; see, e.g. Ref. 13. For we could describe the intermediate solutions based on the so-called comparison, in the case of the electroweak phase transition, please consult. ${ }^{\mathrm{b}}$ In the framework which we consider, "pasted" or "patched" domain walls when the majority of the false vacua get first eliminated - but how it would evolve from there and how long it would evolve still uncertain. See the discussions given later.

The major result of this presentation is that the latent heat (or latent energy), which turns out to be identified as the "bag constant," is huge compared to the radiation density at the cosmological QCD phase transition (i.e. at about $3 \times 10^{-5} \mathrm{~s}$ ). As time evolved to the present, we assume naturally that the percentage of this quantity becomes probably the majority of dark matter (25\% of the composition of the present universe).

\section{The Basics}

A prevailing view regarding our universe is that it originates from the joint making of Einstein's general relativity and the cosmological principle while the observed anisotropies associated with the cosmic microwave background (CMB), the sizes up to about one part in 100,000, might stem, e.g. from quantum fluctuations in the inflation era. In what follows, we wish to first outline very briefly a few key points in the standard scenario so that we shall have a framework which we may employ to elucidate the roles of phase transitions in the early universe.

Based upon the cosmological principle which state that our universe is homogeneous and isotropic, we use the Robertson-Walker metric to describe our universe: ${ }^{16}$

$$
d s^{2}=d t^{2}-R^{2}(t)\left\{\frac{d r^{2}}{1-k r^{2}}+r^{2} d \theta^{2}+r^{2} \sin ^{2} \theta d \phi^{2}\right\} .
$$

\footnotetext{
${ }^{\mathrm{b}}$ For example, see Refs. 14 and 15.
} 
Here the parameter $k$ describes the spatial curvature with $k=+1,-1$ and 0 referring to an open, closed and flat universe, respectively. The scale factor $R(t)$ describes the size of the universe at time $t$.

To a reasonable first approximation, the universe can be described by a perfect fluid, i.e. a fluid with the energy-momentum tensor $T^{\mu}{ }_{\nu}=\operatorname{diag}(\rho,-p,-p,-p)$ where $\rho$ is the energy density and $p$ the pressure. Thus, the Einstein equation, $G^{\mu}{ }_{\nu}=8 \pi G_{N} T^{\mu}{ }_{\nu}+\Lambda g^{\mu}{ }_{\nu}$, gives rise to only two independent equations, i.e. from $(\mu, \nu)=(0,0)$ and $(i, i)$ components:

$$
\begin{aligned}
\frac{\dot{R}^{2}}{R^{2}}+\frac{k}{R^{2}} & =\frac{8 \pi G_{N}}{3} \rho+\frac{\Lambda}{3}, \\
2 \frac{\ddot{R}}{R}+\frac{\dot{R}^{2}}{R^{2}}+\frac{k}{R^{2}} & =-8 \pi G_{N} p+\Lambda .
\end{aligned}
$$

Combining with the equation of state (EOS), i.e. the relation between the pressure $p$ and the energy density $\rho$, we can solve the three functions $R(t), \rho(t)$, and $p(t)$ from the three equations. Further, the above two equations yields

$$
\frac{\ddot{R}}{R}=-\frac{4 \pi G_{N}}{3}(\rho+3 p)+\frac{\Lambda}{3},
$$

showing either that there is a positive cosmological constant or that $\rho+3 p$ must be somehow negative, if the major conclusion of the Supernovae Cosmology Project is correct, ${ }^{17,18}$ i.e. the expansion of our universe still accelerating $(\ddot{R} / R>0)$.

Assuming a simple equation of state, $p=w \rho$, we obtain, from Eqs. (2) and (3),

$$
2 \frac{\ddot{R}}{R}+(1+3 w)\left(\frac{\dot{R}^{2}}{R^{2}}+\frac{k}{R^{2}}\right)-(1+w) \Lambda=0,
$$

which is applicable when a particular component dominates over the others such as in the inflation era (before the hot big bang era), the radiation-dominated universe (e.g. the early stage of the hot big bang era), and the matter-dominated universe (i.e. the late stage of the hot big bang era, before the dark energy sets in to dominate everything else). In light of cosmological QCD phase transition, we would like to examine if the radiation-dominate universe and the matter-dominated universe could ever exist at all, since this has become a dogma in the thinking of our universe.

For the Inflation Era, we could write $p=-\rho$ and $k=0$ (for simplicity), so that

$$
\ddot{R}-\frac{\dot{R}^{2}}{R}=0,
$$

which has an exponentially growing, or decaying, solution $R \propto e^{ \pm \alpha t}$, compatible with the so-called "inflation" or "big inflation." In fact, considering the simplest case of a real scalar field $\phi(t)$, we have

$$
\rho=\frac{1}{2} \dot{\phi}^{2}+V(\phi), \quad p=\frac{1}{2} \dot{\phi}^{2}-V(\phi),
$$


so that, when the "kinetic" term $\frac{1}{2} \dot{\phi}^{2}$ is negligible, we have an equation of state, $p \sim-\rho$. In addition to its possible role as the "inflaton" responsible for inflation, such field has also been invoked to explain the accelerating expansion of the present universe, as dubbed as "quintessence" or "complex quintessence." 19,20

Let us look at the standard textbook argument leading to the radiationdominated universe and the matter-dominated universe:

For the Radiation-Dominated Universe, we have $p=\rho / 3$. For simplicity, we assume that the curvature is zero $(k=0)$ and that the cosmological constant is negligible $(\Lambda=0)$. In this case, we find from Eq. (5):

$$
R \propto t^{\frac{1}{2}}
$$

Another simple consequence of the homogeneous model is to derive the continuity equation from Eqs. (2) and (3):

$$
d\left(\rho R^{3}\right)+p d\left(R^{3}\right)=0 .
$$

Accordingly, we have $\rho \propto R^{-4}$ for a radiation-dominated universe $(p=\rho / 3)$ while $\rho \propto R^{-3}$ for a matter-dominated universe $(p \ll \rho)$. The present universe is believed to have a matter content of about $5 \%$, or of the density of about $5 \times 10^{-31} \mathrm{~g} / \mathrm{cm}^{3}$, much bigger than its radiation content $5 \times 10^{-35} \mathrm{~g} / \mathrm{cm}^{3}$, as estimated from the $3^{\circ}$ blackbody radiation. However, as $t \rightarrow 0$, we anticipate $R \rightarrow 0$, extrapolated back to a very small universe as compared to the present one. Therefore, the universe is necessarily dominated by the radiation during its early enough epochs.

For the radiation-dominated early epochs of the universe with $k=0$ and $\Lambda=0$, we could deduce, also from Eqs. (2) and (3),

$$
\rho=\frac{3}{32 \pi G_{N}} t^{-2}, \quad T=\left\{\frac{3 c^{2}}{32 \pi G_{N} a}\right\}^{\frac{1}{4}} t^{-\frac{1}{2}} \cong 10^{10} t^{-1 / 2}\left({ }^{\circ} \mathrm{K}\right) .
$$

These equations tell us a few important times in the early universe, such as $10^{-11} \mathrm{~s}$ when the temperature $T$ is around $300 \mathrm{GeV}$ during which the EW phase transition is expected to occur, or somewhere between $10^{-5} \mathrm{~s}(\cong 300 \mathrm{MeV})$ and $10^{-4} \mathrm{~s}$ $(\cong 100 \mathrm{MeV})$ during which quarks and gluons undergo the QCD confinement phase transition.

For the Matter-Dominated Universe, we have $p \approx 0$, together with the assumption that $k=0$ and $\Lambda=0$. Equation (5) yields

$$
R \propto t^{\frac{2}{3}}
$$

As mentioned earlier, the matter density $\rho_{m}$ scales like $R^{-3}$, or $\rho_{m} \propto t^{-2}$, the latter similar in the radiation-dominated case.

When $t=10^{9} \mathrm{~s}$, we have $\rho_{\gamma}=6.4 \times 10^{-18} \mathrm{gm} / \mathrm{cm}^{3}$ and $\rho_{m}=3.2 \times$ $10^{-18} \mathrm{gm} / \mathrm{cm}^{3}$, which are close to each other and it is almost near the end of the radiation-dominated universe. The present age of the universe is 13.7 billion years - for a large part of it, it is matter-dominated although now we have plenty of dark energy $(65 \% \sim 70 \%)$. 
However, it is generally believed that our present universe is already dominated by the dark energy (the simplest form being of the cosmological constant; about $70 \%$ ) and the dark matter (about 25\%). The question is when this was so - when the dark part became dominant.

There is another basic point - trivial but important. For both the electroweak and QCD phase transitions in the early universe, if the phase transitions are described (approximately) by the complex fields $\phi$, then the density of the system is given by

$$
\rho=\rho_{\phi}+\rho_{\gamma}+\rho_{m}+\cdots,
$$

before or after or during the phase transition is being taking place. For the electroweak or QCD phase transition, we know that $\rho_{m} \ll \rho_{\gamma}$, but the role played by $\rho_{\phi}$ is clearly of importance in our considerations.

What would be missing in the standard textbook argument? There are two important points: first, the Einstein equation is classical — where would the quantum principle enter? Presumably, we all believe that our universe grew from a tiny bit but physicists are struggling for a hundred years that these tiny objects obey the quantum principle. Second, a more humble request, how do we know, for example, that our universe was once dominated by matter or was in the other epoch once dominated by radiation? The worry comes from two reasons: the dark matter nowadays occupies $25 \%$ of the universe, much larger than the (ordinary) matter (5\%) and there is no reason that dark matter component would disappear suddenly. The other reason is what we will try to explain - maybe the latent heat released in the cosmological QCD or EW phase transition is so much compared to radiation at the point.

We would come back in Sec. 6 to the last point (on the latent heat or energy in the cosmological QCD phase transtion), after we set up the general framework and have gained enough of insights. The crucial point is whether cosmological QCD phase transition is the first-order phase transition - if it is, there is the latent "heat" or energy released in the transition; the story would change dramatically if the amount of energy density turns out to be greater than either $\rho_{\gamma}$ or $\rho_{m}$ in the previous radiation-dominated or matter-dominated era. We have to question what happened if $\rho_{\gamma}$ would no longer be dominant in the radiation-dominated universe the "new" dominant sector of the universe may not influence the "old" radiationdominated piece but Einstein equation in principle no longer guarantee its validity (about the old, no-longer-dominant sector).

\section{The Cosmological QCD Phase Transition}

Let us try to focus on the QCD phase transition in the early universe, or on the cosmological QCD phase transition. 
At the temperature $T>T_{c} \sim 150 \mathrm{MeV}$, i.e. before the phase transition takes place, free quarks and gluons can roam anywhere. As the universe expands and cools, eventually passing the critical temperature $T_{c}$, the bubbles nucleate here and there. These bubbles "explode," as we call it "exploding solitons." When it reaches the "supercooling" temperature, $T_{s}$, or something similar, the previous bubbles become too many and in fact most of them become touched each other - now the false vacua or "bubbles" of different kind (where quarks and gluons can move freely) start to collapse — or we call it "imploding solitons." When all these bubbles of different kind implode completely, the phase transition is now complete.

There is some specialty regarding the QCD phase transition in the early universe. Namely, the collapse of the false vacuum does depend on the inside quarkgluon content - e.g. if we have a three-quark color-singlet combination inside, the collapse of the false vacuum would stop (or stabilize) at a certain radius (we called the bag radius, like in the MIT bag radius); of course, there are meson configurations, glueballs, hybrids, six-quark or multiquark configurations, etc. The QCD phase transition in the early universe does not eliminate all the false vacua; rather, the end state of the transition could have at least lots of baryon or meson states, each of them has some false vacuum to stabilize the system.

How big can a bubble grow? It is with the fastest speed which the bubble can grow is through the speed of light or close to the speed of light. The bubble could sustain from the moment it creates, say, $T \approx T_{c}$ to the moment of supercooling, $T_{s} \sim 0.95 \cdot T_{c}$, or during the time span $t \sim 3 \times 10^{-5} \times 0.05 \mathrm{~s}\left(\right.$ or $\left.1.5 \times 10^{-7} \mathrm{~s}\right)$. So, the bubble can at most grow into $c \cdot 1.5 \times 10^{-7} \mathrm{~s}$ or $4.5 \times 10^{3} \mathrm{~cm}$.

How big was the universe during the cosmological QCD phase transition? Compared to the size now, an estimate is the expansion of $5.7 \times 10^{12}$, a huge factor. (See the beginning of Sec. 6.) In the simplest approximation (when the scalar fields do not couple to the other fields, such as gluons or quarks), the domain walls could not disappear - not only sometime because of the possible nontrivial topology but that there should eventually be some QCD dynamics to annihilate the walls. So, in light of the huge expansion factor, the domain wall structure cannot survive, except the strict topology (which we call "domain-wall nuggets").

As a yardstick, we note that, at $t \sim 10^{-5} \mathrm{~s}$ or $T \sim 300 \mathrm{MeV}$, we have

$$
\rho_{\gamma} \sim 6.4 \times 10^{10} \mathrm{gm} / \mathrm{cm}^{3}, \quad \rho_{m} \sim 3.2 \times 10^{3} \mathrm{gm} / \mathrm{cm}^{3} .
$$

Or, at $t \sim 3.30 \times 10^{-5} \mathrm{~s}$ or $T=T_{c} \sim 150 \mathrm{MeV}$, we have

$$
\rho_{\gamma}=5.88 \times 10^{9} \mathrm{gm} / \mathrm{cm}^{3}, \quad \rho_{m}=6.51 \times 10^{2} \mathrm{gm} / \mathrm{cm}^{3} .
$$

Slightly later when QCD phase transition has completed, at $t \sim 10^{-4}$ s or $T \sim$ $100 \mathrm{MeV}$, we have

$$
\rho_{\gamma} \sim 6.4 \times 10^{8} \mathrm{gm} / \mathrm{cm}^{3}, \quad \rho_{m} \sim 1.0 \times 10^{2} \mathrm{gm} / \mathrm{cm}^{3} .
$$


In what follows, we use the so-called "bag models" " to have the simplified version of quark confinement - I think it is important to use the simplified version in the complicated cosmological environment, in order to extract meaningful results.

When the low-temperature bubbles start to show up (i.e. to nucleate), it is about $T_{c} \approx T<T_{s}$. This period is to be called "exploding soliton era," $24-27$ to be described in the next section (Sec. 4). The supercooling temperature $T_{s}$, presumably $\sim 0.95 T_{c}$ (to be determined more reliably in the future), refers to the situation where the bubbles begin to saturate. We call it the "colliding soliton era." This is to be described in Sec. 5. Presumably it would be over when $T \leq T_{s}-\left(T_{c}-T_{s}\right)$ or longer. So, the cosmological QCD phase transition would be over when the universe was $10^{-4} \mathrm{~s}$ old. The important things is that, because the phase transition is of the first-order, it releases a huge amount of energy:

$$
\rho_{\mathrm{vac}}=1.0163 \times 10^{14} \mathrm{gm} / \mathrm{cm}^{3},
$$

clearly much bigger than the radiation density (cf. Eq. (14)). This quantity is in fact the same as "the zero-point energy." That is why we question the radiationdominated universe.

When the low-temperature bubbles fill up the space, the neighboring two bubbles would in general be labeled by different $\theta_{i, j}$ representing different but degenerate vacua — we assume that there are infinite many choices of $\theta$; they are degenerate but complete equivalent. The domain wall is used to separate the two regions. Three different regions would meet in a line - which we call a vortex. We have to estimate the total energy associated with the domain walls and the vortices - particularly when these objects persist to live on for a "long" time say, $\tau \gg 10^{-4} \mathrm{~s}$. These domain walls and vortices are governed, in the QCD phase transition in the early universe, by the QCD dynamics - this is an important point; if not, what else? It is a tough question to figure out how long the universe would stabilize itself through QCD dynamics and others; my rough guess is from a few seconds to years, say $\tau$.

For the moment, QCD enables us to make some estimates. Let us focus on $t \sim 10^{-4} \mathrm{~s}$, where $\rho_{m}=1.0 \times 10^{2} \mathrm{gm} / \mathrm{cm}^{3}$. Or, considering a unit volume of $1.0 \mathrm{~cm}^{3}$, the amount of the matter would be $100 \mathrm{gm}$ or $5.609 \times 10^{31} \mathrm{GeV} / c^{2}$. One proton or neutron weighs about $1 \mathrm{GeV} / c^{2}$ so, in a volume $1.0 \mathrm{~cm}^{3}$ at $t \sim 10^{-4} \mathrm{~s}$, we had at least $5.609 \times 10^{31}$ baryons or, in the MIT bag model language, $5.609 \times 10^{31}$ bags or $R=1.0$ fermi false vacua associated with the system. To begin with, all the excited baryons and mesons, including topological objects, and multibaryons, all have equal opportunities. But, remembering $1 \mathrm{~cm}^{3}=10^{39}$ fermi $^{3}$, most space had to collapse into the true vacua with different $\theta_{i}$. That is, this used to be called "the radiation-dominated era" and so the physics of matter was quite negligible.

When lots of bubbles nucleated, most of which grew rapidly or exploded, then a lot of bubble collisions and eventually turned around, and then the bubbles of false

${ }^{\mathrm{c}}$ References 21 and 22 (for the bag model parameters, see, e.g. Ref. 23). References 24 and 25 (for generalizations, see, e.g. Refs. 26 and 27$)$. 
vacuum imploded, these things might happen without the meaning of the "local" temperatures - it is likely that the notion of equilibrium or near-equilibrium could lose the meaning. We believe that, at these moments, the dynamics in terms of scalar fields or QCD remains to be valid and applicable. To describe the situation in terms of the effective scalar fields, we simplify the genuine QCD dynamics but eventually we have to take into account the couplings between these scalar fields and the gluons/quarks. These couplings in turn determine the timings of these processes.

\section{Exploding Solitons}

(Note: A simplified version of this section, and the following two sections, was presented during my 30-min. talk. Here I have included it for the sake of completeness. The materials are also presented in my talk at 10th Asia-Pacific Physics Conference and in Korea Journal of Physics (to be published).)

We begin our study by examining an isolated bubble - expanding, that is, inside the bubble, it is the true vacuum labeled by some $\theta$; outside the bubble, the false vacuum; we are thinking of the universe cooling down and expand. That is, how the bubble nucleates in the false vacuum (high-temperature). Remember that this happened in the period of time roughly when $T_{c} \leq T \leq T_{s}$.

Consider a spherical wall of radius $R$ and thickness $\Delta$ separating the true vacuum inside from the false vacuum outside. The energy density difference of the vacua is $B$, the bag constant in the most simplified situation, and the energy $\tau$ per unit area associated with the surface tension on the separating wall is a quantity to be calculated but nevertheless is small compared to the latent heat. If the wall expands outward for a distance $\delta R$, then the energy budget arising from the vacuum change is

$$
B \cdot 4 \pi R^{2} \cdot \delta R-\tau \cdot 4 \pi\left\{(R+\delta R)^{2}-R^{2}\right\}=-p \delta V,
$$

where $p$ is the pressure and is so defined that a negative pressure would push the wall outward. (We use the notation $\tau$ here, since $\sigma$ and $\rho$ are reserved for other purposes.)

When the surface tension energy required for making the wall bigger is much less than the latent heat required from the expansion of the bubble, the bubble of the stable vacuum inside will grow in an accelerating way, possibly resulting in explosive growth of the bubble. The scenario may be as follows: when the universe expands and cools, to a temperature slightly above the critical temperature $T_{c}$, bubbles of lower vacua will nucleate at the spots where either the temperature is lower, and lower than $T_{c}$, or the density is higher, and higher than the critical density $\rho_{c}$. As the universe continues to expand and cool further, most places in the universe have the temperature slightly below $T_{c}$; that is, the destiny arising from eternal expansion of the universe is driving the average temperature of the entire universe toward below the critical temperature. The universe must find a way to convert itself entirely into another vacuum, the true vacuum at the lower temperature. 
Maybe we should add a footnote. We are afraid that the situation is much more complicated - the local temperature may lose its meaning; in other words, there are too many things happening at a spot in a short time interval so that nonequilibrium is the real situation. Thus, the above statements regarding the "temperature" are for reference only.

Therefore, we have a situation in which bubbles of true vacua pop up (nucleate) here and there, now and then, and each of them may grow explosively in the environment made of the false vacuum for now, but previously the true vacuum when the temperature was still near the critical temperature $T_{c}$. In the expanding universe which cooled down relatively rapidly, i.e. from $T_{c}$ to the supercooling temperature $T_{s}$, the situation is awfully complicated. When the temperature becomes lower than $T_{s}$, the problem can be modeled, in the simplest way, by characterizing the vacuum structure by a complex scalar field interacting via the potential $V(\phi)$ :

$$
V(\phi)=\frac{\mu^{2}}{2} \phi^{*} \phi+\frac{\lambda}{4}\left(\phi^{*} \phi\right)^{2}, \quad \mu^{2}<0, \quad \lambda>0
$$

For $T>T_{c}$, we have $\mu^{2}(T)>0$ and $\lambda>0$, so it is between $T_{c}$ and $T_{s}$ when the situations are awfully complicated (and we try to avoid in this paper). Note also that, in the complex scalar field description, the true vacua have degeneracy described by a continuous real parameter $\theta . \phi=0$ everywhere in the space-time describes the false vacuum for the universe at a temperature below the critical temperature $T_{c}$. Consider the solution for a bubble of true vacuum in this environment. It is required that the field $\phi$ must satisfy the field equation everywhere in space-time, including crossing the wall of thickness $\Delta$ to connect smoothly the true vacuum inside and the false vacuum outside. This is why we may call the bubble solution "a soliton," in the sense of a nontopological soliton of T. D. Lee's. However, the soliton grows in an accelerating way, or the name "exploding soliton."

Of course, it is much easier to describe the situation in terms of a real scalar field in view of no degeneracy. Our solutions could easily be extended to the real scalar field case.

The situation must have changed so explosively that at a very short instant later the universe expands even further and cools to even a little more farther away from $T_{c}$ and most places in the universe must be in the true vacuum, making the previously false vacuum shrink and fractured into small regions of false vacua, presumably dominantly in spherical shape, which is shrinking in an accelerating way, or "implosively." Using again the complex scalar field as our language, we then have "imploding solitons."

In what follows, we attempt to solve the problem of an exploding soliton, assuming that the values of both the potential parameters $\mu^{2}$ and $\lambda$ are fairly stable during the period of the soliton expansion. The scalar field must satisfy:

$$
\frac{1}{r^{2}} \frac{\partial}{\partial r}\left(r^{2} \frac{\partial \phi}{\partial r}\right)-\frac{\partial^{2} \phi}{\partial t^{2}}=V^{\prime}(\phi) .
$$


The radius of the soliton is $R(t)$ while the thickness of the wall is $\Delta$ :

$$
\begin{gathered}
\phi=\phi_{0} \quad \text { for } \quad r<R_{0}+v t-\frac{\Delta}{2}, \\
=0 \quad \text { for } \quad r>R_{0}+v t+\frac{\Delta}{2},
\end{gathered}
$$

with $R(t)=R_{0}+v t$ and $v$ the radial expansion velocity of the soliton.

We may write

$$
\phi \equiv f(r+v t) ; \quad w \equiv\left(1-v^{2}\right) r,
$$

so that the field equation becomes

$$
\frac{d^{2} f}{d w^{2}}+\frac{2}{w} \frac{d f}{d w}=\left(1-v^{2}\right)^{-1} \lambda f\left(|f|^{2}-\phi_{0}^{2}\right) .
$$

We will be looking for a solution of $f$ across the wall so that it connects smoothly the true-vacuum solution inside and the false vacuum solution outside.

Introducing $g \equiv w f(w)$, we find

$$
g^{\prime \prime}=\left(1-v^{2}\right)^{-1} \lambda g\left\{\left|\frac{g}{w}\right|^{2}-\phi_{0}^{2}\right\},
$$

an equation which we may solve in exactly the same manner as the colliding-wall problem to be elucidated in the next section.

We could examine the issue whether we could treat the QCD phase transition as the first-order transition. The basic equation as revealed by Eq. (17) indicates that, if the pressure term is outward, the latent heat (i.e. the bag constant term) must be there - note that the sign of the surface tension is also opposite. That is, if the phase transition is not first-order, the pressure would be outward negative - the bubbles would be in general shrinking - a strange result, indeed! In other words, it is in fact nontrivial to think of the QCD phase transition as not first-order.

\section{Colliding Walls: Formation of "Pasted" Domain Walls}

When bubbles of true vacua grow explosively, the nearby pair of bubbles will soon squeeze or collide with each other, resulting in merging of the two bubbles while producing cosmological objects that have specific coupling to the system. The situation is again extremely complicated. Remember that this happened when $T \sim T_{s}$, not too long after.

We try to disentangle the complexities by looking at between the two bubble walls that are almost ready to touch and for the initial attempt neglecting the coupling of the vacuum dynamics to the matter content. Between the two bubble walls, especially between the centers of the two bubbles, it looks like a problem of plane walls in collision - and this is where we try to solve the problem to begin with.

In fact, we have to consider one bubble first - the spherical situation as in the previous section but the bubble is "very" large we could look at the $z$-direction 
in the sufficiently good plane approximation (i.e. all bubble surfaces are just like planes). At this point, we have one wall, with thickness $\Delta$, moving with velocity $v$ in the $z$ direction; on the left of the wall is the false vacuum, and on the right the true vacuum.

The wall, of thickness $\Delta$, separates the true vacuum on one side from the false vacuum on the other side of the wall. For the sake of simplicity, the wall is assumed parallel to the $(x y)$-plane and are infinite in both the $x$ and $y$ directions. In addition, at some instant the wall is defined between $z=z_{0}-\frac{\Delta}{2}$ and $z=z_{0}+\frac{\Delta}{2}$ with the instantaneous velocity $+v$; this region connects the true vacuum with the false vacuum.

In other words, we need to consider the case that we have two parallel walls approaching each other: the left-hand one at some instant is at $-R-\frac{\Delta}{2}<z<$ $-R+\frac{\Delta}{2}$ while the right-hand one at $R-\frac{\Delta}{2}<z<R+\frac{\Delta}{2}$; in the middle $-R+\frac{\Delta}{2}<$ $z<+R-\frac{\Delta}{2}$, it is the false vacuum; the walls are moving toward each other, so the false vacuum gets squeeze out.

For $z>R+\frac{\Delta}{2}$ and all $x$ and $y$, the complex scalar field $\phi$ assumes $\phi_{0}$, a value of the true vacuum (the ground state). On the other hand, for $-R+\frac{\Delta}{2}<z<+R-\frac{\Delta}{2}$ and all $x$ and $y$, the complex scalar field $\phi$ assumes $\phi=0$, the false vacuum. As indicated earlier, the field $\phi$ must satisfy the field equation everywhere in spacetime:

$$
\frac{\partial^{2} \phi}{\partial z^{2}}-\frac{\partial^{2} \phi}{\partial t^{2}}=V^{\prime}(\phi) .
$$

We may write the wall on the right-hand side but moving toward the left with the velocity $v$ :

$$
\phi=f(z-v t) \quad \text { for } \quad z-v t>0, \quad t<R / v
$$

so that

$$
\left(1-v^{2}\right) f^{\prime \prime}=\lambda f\left(|f|^{2}-\sigma^{2}\right), \quad \sigma \equiv\left|\phi_{0}\right|>0 .
$$

In fact, we are interested in the situation that the function $f$ is complex:

$$
f \equiv u e^{i \theta}
$$

so that, with $\tilde{\lambda} \equiv \lambda /\left(1-v^{2}\right)$,

$$
\begin{gathered}
u^{\prime \prime}-u\left(\theta^{\prime}\right)^{2}=\tilde{\lambda} u\left(u^{2}-\sigma^{2}\right), \\
2 u^{\prime} \theta^{\prime}+u \theta^{\prime \prime}=0
\end{gathered}
$$

Integrating the second equation, we find

$$
u^{2} \theta^{\prime}=K \text {, }
$$

with $K$ an integration constant. The equation for $u$ is thus given by

$$
u^{\prime \prime}=\frac{K}{u^{3}}+\tilde{\lambda} u\left(u^{2}-\sigma^{2}\right),
$$


provided that the $\theta$ function is defined (in the region of the true vacuum and the wall).

Let us try to focus on the last two basic equations - for $u$ and $\theta$, say, as the functions of $\xi$ (e.g. $\xi=z \pm v t$ ). For $\xi \geq \Delta$, we have $\phi=\sigma e^{i \theta}$ (the true vacuum) and, for $\xi<0$, we have $\phi=0$ (the false vacuum; with $\theta$ undetermined). We find, for $\xi \rightarrow 0^{+}$,

$$
\theta=\frac{1}{2} \sqrt{-K}(\ln \xi)(1+F(\xi))+C_{0},
$$

with $C_{0}$ a constant and $F(\xi)$ regular near $\xi \sim 0$. Therefore the $\theta(\xi)$ function could be "mildly singular" or blow up near $\xi \sim 0$ - this is in fact a very important point.

Of course, the equation for $u$ can be integrated out to obtain the result. For the "wall" region (i.e. $0<\xi<\Delta$ ), the solution reads as follows:

$$
\xi=\frac{\sigma^{2}}{2} \int_{0}^{u^{2} / \sigma^{2}} \frac{d y}{\sqrt{-K+\alpha y-2 \beta y^{2}+\beta y^{3}}},
$$

with

$$
\Delta=\frac{\sigma^{2}}{2} \int_{0}^{1} \frac{d y}{\sqrt{-K+\alpha y-2 \beta y^{2}+\beta y^{3}}} .
$$

Here $\beta \equiv \frac{\tilde{\lambda}}{2} \sigma^{6}$, and $K$ and $\alpha$ parameters related to the integration constants. Of course, the solution in true-vacuum region can be obtained by extension.

In the wall region, we could compute the surface energy per unit area (i.e. surface tension mentioned earlier in Eq. (17)):

$$
\tau=\int_{0}^{\Delta} d \xi \frac{1}{2}\left\{\left(u^{\prime}\right)^{2}+u^{2}\left(\theta^{\prime}\right)^{2}\right\}
$$

some integral easy to calculate.

There is an important note - that is, the solution for $\phi$ obtained so far applies for the true vacuum and the wall, and which is continuous in the region; how about the false vacuum? This is an important question because in the false vacuum we know that $u=0$ but $\theta$ is left undetermined. So, in first-order phase transitions we have certain function undefined in the false-vacuum region(s). This is a crucial point to keep in mind with.

As a parenthetical footnote, we note that the equation for the exploding or imploding spherical soliton, Eq. (22), may be integrated and solved in an identical manner.

Now let us focus on the merge of the two bubbles - the growing of the two true-vacuum bubbles such that the false-vacuum region gets squeezed away. This is another difficult dynamical question. In fact, we can make the false-vacuum region approaching to zero, i.e. the region with the solution $u=0$ gets squeezed away; one true-vacuum region with $\theta_{1}$ and $\Delta_{1}$ (the latter for the wall) is connected with the one with $\theta_{2}$ and $\Delta_{2}$ - we could use $\left(K_{1}, K_{2}\right)$ to label the new boundary; to be precise, we could call it "the pasted domain wall" or "the patched domain wall." It is in fact 
two walls pasted together - if we look at the boundary condition in between, we realize that the structure would persist there for a while to go. The pasted domain wall could evolve further but this may not be relevant for counting the energies involved. The evolved forms of the pasted domain walls could be determined by the topology involved - for the purpose of this paper, we can ignore this fine aspect.

Suppose that the cosmological QCD phase transition was just completed - we have to caution that, not everywhere, the false vacua be replaced by the true vacua so that in between the walls be replaced (approximately) by the pasted domain walls. There are places for color-singlet objects (i.e. hadrons) which quarks and gluons tried to hide; these places are still called by the "false vacua" with the volume energies. Thus, the volume energy, i.e. $B$ in Eq. (17) or defined suitably via $\lambda$ and $\mu^{2}$ (in Eq. (18)), or at least some portion of it, may convert itself into the surface energy and others $-B=57 \mathrm{MeV} / \mathrm{fm}^{3}$ using the so-called "bag constant" in the MIT bag model ${ }^{21-23}$ or Columbia bag model. ${ }^{24-27}$

This energy density $B=57 \mathrm{MeV} / \mathrm{fm}^{3}=1.0163 \times 10^{14} \mathrm{gm} / \mathrm{cm}^{3}$ is huge as compared to the radiation density $\rho_{\gamma}$ (which is much bigger than the matter density $\rho_{m}$ ) at that time, $t \sim 10^{-5} \sim 10^{-4} \mathrm{~s}$ (see Eqs. (13)-(15)). Some exercise indicates that this quantity of energy is exactly the latent "heat" or energy released in the first-order phase transition.

The cosmological QCD phase transition should leave its QCD mark here - since the volume energy that stays with the "false vacuum" is simply reduced because the volumes with the "false vacua" are greatly reduced — but not eliminated because quarks and gluons, those objects with colors, still have some places to go (or, to hide themselves).

\section{Possible Connection with the Dark Matter}

Let us begin by making a simple estimate - the expansion factor since the QCD phase transition up to now. The present age of the universe is 13.7 billion years or $13.7 \times 10^{9} \times 365.25 \times 24 \times 3600$ or $4.323 \times 10^{17}$ s. As indicated earlier (cf. the end of Sec. 2), about the first $10^{9} \mathrm{~s}$ period of the hot big bang is previouslybelieved radiation-dominated. Consider the length 1.0 fermi at $t \sim 10^{-5} \mathrm{~s}$, it will be expanded by a factor of $10^{7}$ up to $t \sim 10^{9} \mathrm{~s}$ (radiation-dominated) and expanded further by another factor of $5.7 \times 10^{5}$ until the present time - so, a total expansion factor of $5.7 \times 10^{12}$; changing a length of 2 fermi at $t \sim 10^{-5} \mathrm{~s}$ into a distance of $1 \mathrm{~cm}$ now. A proton presumably of $R=1$ fermi at $t \sim 10^{-4}$ s should be more or less of the same size now; or, the bag constant or the energy associated with the false vacuum should remain the same.

What would happen to the pasted or patched domain walls as formed during the cosmological QCD phase transition? According to Eqs. (30) and (31) together with Eq. (32), we realize that the solutions in previously two different true-vacuum regions cannot be matched naturally - unless the $K$ values match accidently. On 
the other hand, it is certain that the system cannot be stretched or over-stretched by such enormous factor, $10^{12}$ or $10^{13}$.

As we said earlier, at some point after the supercooling temperature $T_{s}$, say, at $T_{s}-\lambda\left(T_{c}-T_{s}\right)$ (with $\lambda$ an unknown factor, presumably $\lambda \gg 1$ ), the system (the universe) was temporarily stabilized since most of the pasted or patched domain walls had no where to go. Remember that all these happened in a matter of a fraction of $10^{-4} \mathrm{~s}$, as judging from the size of $T_{c}$ and $T_{s}$. The next thing to happen is probably the following.

We believe that the field $\phi$, being effective, cannot be lonely (i.e. noninteracting with other fields); in fact, we believe that there are higher-order interactions such as

$$
c_{0} \phi G_{\mu}^{a} G^{\mu, a}, \quad c_{1} \phi G G G, \ldots, d_{0} \phi \bar{\psi} \psi,
$$

some maybe being absent because of the nature of $\phi$. The sizes of these couplings determine the time scales needed for these interactions. In other words, we may believe that the strong interactions are primarily responsible for the phase transition in question, such that the effective field $\phi$ couples to the gluon and quark fields; the details of the coupling are subject to investigations.

That is, when the field $\phi$ responsible for the pasted or patched domain walls is effective - the $\phi$ field couples, in the higher-order (and thus weaker) sense, to the gluon and quark fields. It is very difficult to estimate what time is needed for pasted domain walls to disappear, if there are no nontrivial topology involved. If there is some sort of nontrivial topology present, there should left some kind of topological domain nugget - however, energy conservation should tell us that it cannot be expanded by too many orders (but our universe did expand for many many orders of magnitude). I would guess that it takes about from a fraction of a second to several years (from the strong interaction nature of the problem), but certainly before the last scattering surface (i.e. $3.79 \times 10^{5}$ years).

To summarize, the energy associated with the cosmological QCD phase transition, mainly the vacuum energy associated with the false vacuum, disappeared in several ways, viz.: (1) the bag energies associated with the baryons and all the other color-singlet objects, (2) the energies with all kinds of topological domain nuggets or other topological objects, and (3) the decay products from pasted or patched domain walls with trivial topology.

Let us begin with the critical temperature $T=T_{c} \approx 150 \mathrm{MeV}$ or $t \approx 3.30 \times$ $10^{-5}$ s. At this moment, we have

$\rho_{\text {vac }}=1.0163 \times 10^{14} \mathrm{gm} / \mathrm{cm}^{3}, \quad \rho_{\gamma}=5.88 \times 10^{9} \mathrm{gm} / \mathrm{cm}^{3}, \quad \rho_{m}=6.51 \times 10^{2} \mathrm{gm} / \mathrm{cm}^{3}$.

Here the first term is what we expect the system to release - the so-called "latent heat;" I call it "latent energy" for obvious reasons. The identification of the latent "heat" with the bag constant is well-known in Coulomb bag models. ${ }^{24-27}$ 
This can be considered just before the cosmological QCD phase transition which took place - at the moment the energy components which we should take into consideration.

As time went on, the universe expanded and the temperature cooled further from the critical temperature to the supercooling temperature $\left(T_{s} \sim 0.95 \times T_{c}\right.$ with the fraction 0.95 in fact unknown) and even lower, and then the cosmological QCD phase transition was complete. When the phase transition was complete, we should estimate how the energy $\rho_{\text {vac }}$ is to be divided.

Let us assume that the QCD phase transition was completed at the point $T_{s}$ (in fact maybe a little short after $T_{s}$ ). Let us take $T_{s}=0.95 T_{c}$ for simplicity. We would like to know how the energy $\rho_{\text {vac }}$ is to be divided. First, we can estimate those remained with the baryons and other color-singlet objects - the lower limit is given by the estimate on the baryon number density (noting that one baryon weighs about $\left.1.0 \mathrm{GeV} / c^{2}\right)$ :

$\rho_{m}=6.51 \times 10^{2} \mathrm{gm} / \mathrm{cm}^{3} \times 0.5609 \times 10^{24} \mathrm{GeV} / c^{2} / \mathrm{gm}=3.65 \times 10^{26} \mathrm{GeV} / c^{2} / \mathrm{cm}^{3}$.

So, in the volume $1.0 \mathrm{~cm}^{3}$ or $10^{39}$ fermi $^{3}$, we have at least $3.65 \times 10^{26}$ baryons. One baryon has the volume energy (i.e. the bag energy or the false vacuum energy) $57 \mathrm{MeV} /$ fermi $^{3} \times \frac{4}{3} \pi\left(1.0\right.$ fermi) ${ }^{3}$ (which is $238.8 \mathrm{MeV}$ ). So, in the volume $1.0 \mathrm{~cm}^{3}$, we have at least $238.8 \mathrm{MeV} \times 3.65 \times 10^{26}$ or $8.72 \times 10^{25} \mathrm{GeV}$ in baryon bag energy. Or, in different units $8.72 \times 10^{25} /\left(0.5609 \times 10^{24}\right) \mathrm{gm} / c^{2}$ or $155.5 \mathrm{gm} / \mathrm{c}^{2}$. Only a tiny fraction of $\rho_{\mathrm{vac}}$ is to be hidden in baryons or other color-singlet objects after the QCD phase transition in the early universe.

So, where did the huge amount of the energy $\rho_{\text {vac }}$ go? In the beginning of the end of the phase transition, the pasted domain walls with the huge kinetic energies seem to be the main story. A pasted domain wall is forming by colliding two domain walls while eliminating the false vacuum in between. The kinetic energies associated with the previously head-on collision become vibration, center-of-mass motion, etc. Of course, the pasted domain walls would evolve much further such as through the decaying interactions given earlier or forming the "permanent" structures. In any case, the total energy involved is known reasonably - a large fraction of $\rho_{\text {vac }}$, much larger than the radiation $\rho_{\gamma}$ (with $\rho_{m}$ negligible at this point).

The story is relatively simple when the cosmological QCD phase transition was just completed and most "pasted" domain walls still have no time to evolve. We return to Eqs. (2) and (3) (i.e. Einstein equations) for the master equations together with the equation of state with $\rho$ and $p$ determined by the energy-momentum tensor:

$$
T_{\mu \nu}^{\phi}=g_{\mu \alpha} \frac{\partial L}{\partial\left(\partial_{\alpha} \phi\right)} \partial_{\nu} \phi-L g_{\mu \nu} .
$$

Further analysis indicates that the equation of state for the "pasted" or "patched" domain walls is nothing unusual - the reason is that we are working in the real 
four-dimensional space-time and all of the objects are of finite dimensions in all the directions. The "domain walls" discussed by us are for real and cannot be stretched to infinity in a certain dimension.

In fact, there is certain rule which one cannot escape. Let assume a simple equation of state, $\rho=w p$, for simplicity and come to look at Eq. (5). Let us consider the situation in which there is no curvature $k=0$ and the cosmological constant $\lambda$ is not yet important:

$$
2 \frac{\ddot{R}}{R}+(1+3 w) \frac{\dot{R}^{2}}{R^{2}}=0,
$$

which yields

$$
R \propto t^{n}
$$

with $n=\frac{2}{3} \cdot \frac{1}{1+w}$.

From the equation of continuity, $d(\rho R)+p d\left(R^{3}\right)=0$, it is easy to obtain $\rho \propto R^{3(1+w)}$. Thus, we deduce that, under very general situations, the density behaves like

$$
\rho=C t^{-2}
$$

where the constant $C$ is related to $w$ in the simplified equation of state. It is clear that the limit to $w=-1$ (the cosmological constant) is a discontinuity.

Of course, Eq. (4) is still valid:

$$
\frac{\ddot{R}}{R}=-\frac{4 \pi G_{N}}{3}(\rho+3 p)+\frac{\Lambda}{3} .
$$

This has an important consequence - the idea of the previous universe expansion usually based on the radiation alone from $t \sim 10^{-10} \mathrm{~s}$ (after the cosmological electroweak phase transition had taken place) to $t \sim 10^{9} \mathrm{~s}$ (when it was close that $\rho_{\gamma}=\rho_{m}$ ) has to be modified because the latent energy $\rho_{\text {vac }}$ was about $2 \times 10^{5}$ times the radiation energy at the moment of the cosmological QCD phase transition.

Shown in Fig. 1 is our main result - even though it is a qualitative figure but it tells us a lot. At $t \sim 3.30 \times 10^{-5} \mathrm{~s}$, where did the latent energy $10^{14} \mathrm{gm} / \mathrm{cm}^{3}$ evolve into? We should know that the curve for $\rho_{\gamma}$, for massless relativistical particles, is the steepest in slope. The other curve for $\rho_{m}$ is the other limit for matter (which $P \approx 0$ ). In this way, the latent energy is connected naturally with the curve for $\rho_{\mathrm{DM}}$ - in fact, there seems to be no other choice. Remember that $\rho \propto t^{-2}$ except the slope for different types of "matter."

Coming back to Eq. (43) or Eq. (4), we could assume for simplicity that when the cosmological QCD just took place the system follows with the relativistical pace (i.e. $P=\rho / 3$ ) but when the system over-stretched enough and had evolved long enough it was diluted enough and became nonrelativistic (i.e. $P \approx 0$ ). It so happens that in both cases the density to the governing equation, Eq. (43) or Eq. (4), looks like $\rho \propto t^{-2}$ although it is $R \propto t^{\frac{1}{2}}$ followed by $R \propto t^{\frac{2}{3}}$. 


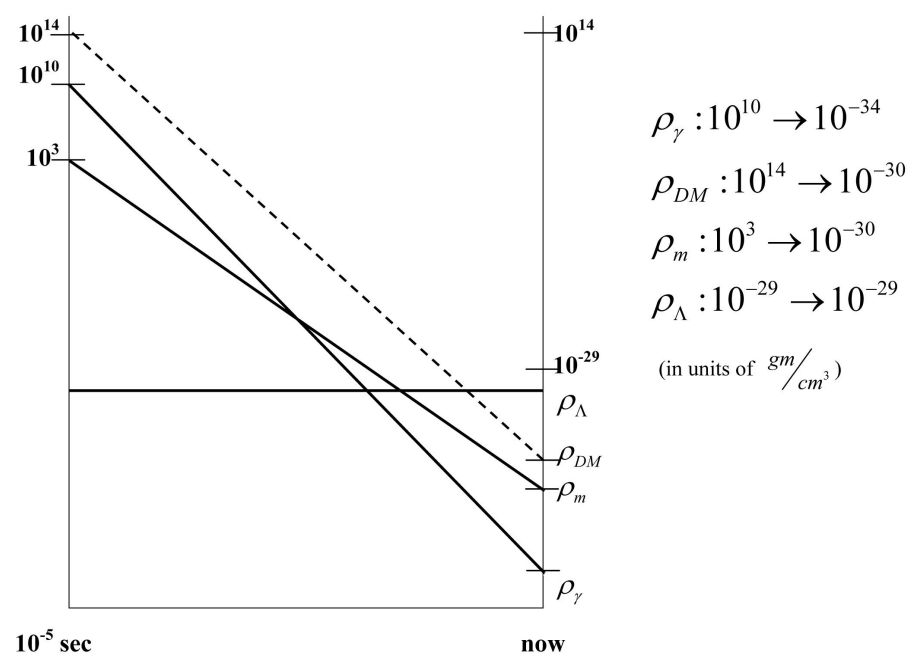

Fig. 1. The various densities of our universe versus time.

It is so accidental that what we call "the radiation-dominated universe" is in fact dominated by the latent energy from the cosmological QCD phase transition in the form of "pasted" or "patched" domain walls and the various evolved objects. In our case, the transition into the "matter-dominated universe," which happened at a time slightly different from $t \sim 10^{9} \mathrm{~s}$, occurred when all the evolutions of the pasted domain walls ceased or stopped. In other words, it is NOT the transition into the "matter-dominated universe," as we used to think of.

In fact, the way of thinking of the "dark matter," or the majority of it, turns out to be very natural. Otherwise, where did the $25 \%$ content of our universe come from? Of course, one could argue about the large amount of the cosmological QCD phase transition. We believe that the curves in Fig. 1 make a lot of sense.

Of course, one should ask what would happen before the cosmological QCD phase transition. It might not be the radiation-dominated. I believe that it opens up a lot of important and basic questions.

\section{Outlook}

To sum up, we tried to illustrate how to describe the QCD phase transition in the early universe, or the cosmological QCD phase transition.

The scenario that some first-order phase transitions may have taken place in the early universe offers us one of the most intriguing and fascinating questions in cosmology. In fact, the role played by the latent "heat" or energy released in the process is highly nontrivial.

In this talk, we assume that the QCD phase transition, which happened at a time $t \approx 3.30 \times 10^{-5} \mathrm{~s}$ or at the temperature of about $150 \mathrm{MeV}$ and accounts for confinement of quarks and gluons to within hadrons in the true QCD vacuum, 
would be of first-order. Thus, it is sufficient to approximate the true QCD vacuum as one of degenerate vacua and when necessary we try to model it effectively via a complex scalar field with spontaneous symmetry breaking. We examine how and how long "pasted" or "patched" domain walls were formed, how and how long such walls evolve further, and why the majority of dark matter might be accounted for in terms of these evolved objects. [It is much easier to examine the case of the real scalar field.]

Our central result could be summarized by Fig. 1 together with the explanations. Mainly, we are afraid that the "radiation-dominated" epoch and the "matterdominated" epoch, in the conventional sense, could not exist once the cosmological QCD phase transition took place. That also explains why there is the $25 \%$ darkmatter content, larger than the baryon content, in our present universe.

In other words, if what we are proposing for the dark matter is largely correct, then at least the "radiation-dominated universe" terminated when the cosmological QCD phase transition took place - from there on, we have something like the "dark-matter-dominated universe." So, it is indeed important to determine whether the QCD phase transition is first-order. ${ }^{8,9}$

\section{Acknowledgments}

The Taiwan CosPA project is funded by the Ministry of Education (89-N-FA01-1-0 up to 89-N-FA01-1-5) and the National Science Council (NSC 95-2752-M-002-007$\mathrm{PAE}$ and others). This research is also supported in part as another National Science Council project (NSC 96-2752-M-002-007-PAE and NSC 96-2112-M-002-023-MY3).

\section{References}

1. C. N. Yang, AAPPS Bulletin 15(1), 4 (2005).

2. G. Smoot et al., Astrophys. J. 396, L1 (1992).

3. C. Bennett et al., Astrophys. J. 396, L7 (1992).

4. E. Wright et al., Astrophys. J. 396, L11 (1992).

5. C. L. Bennett, M. S. Turner and M. White, Physics Today November 32 (1997).

6. See, e.g., the news in Physics Today, April 2003, p. 21; and references therein.

7. See, e.g., the news in Physics Today May 2006, p. 16; and references therein.

8. Y. Aoki, G. Endrodi, Z. Fodor, S. D. Katz and K. K. Szabo, Nature 443, 675 (2006), arXiv:hep-lat/0611014.

9. M. Cheng et al., Phys. Rev. D 75, 034506 (2007), arXiv:hep-lat/0612001.

10. E. Shuryak and T. Schaefer, Annu. Rev. Nucl. Part. Sci. 47, 359 (1997).

11. M. S. Turner, Phys. Rep. 197, 67 (1990).

12. B. Svetitsky and L. G. Yaffe, Nucl. Phys. B 210, 423 (1982).

13. A. Zhitnitsky, arXiv:astro-ph/0603064, 29 July 2006, and references therein.

14. H. Kurki-Suonio and M. Laine, Phys. Rev. Lett. 77, 3951 (1996).

15. R. M. Haas, Phys. Rev. D 57, 7422 (1998).

16. E. W. Kolb and M. S. Turner, The Early Universe (Addison-Wesley, 1994).

17. Supernova Cosmology Project (S. Perlmutter et al.), Astrophys. J. 517, 565 (1999).

18. Supernova Search Team (A. G. Riess et al.), Astron. J. 116, 1009 (1998).

19. R. R. Caldwell, R. Dave and P. J. Steinhardt, Phys. Rev. Lett. 80, 1582 (1998). 
20. J.-A. Gu and W-Y. P. Hwang, Phys. Lett. B 517, 1 (2001).

21. A. Chodos, R. L. Jaffe, K. Johnson, C. B. Thorn and V. F. Weisskopf, Phys. Rev. D 9, 3471 (1974).

22. T. A. DeGrand, R. L. Jaffe, K. Johnson and J. Kiskis, Phys. Rev. D 12, 2060 (1975).

23. W-Y. P. Hwang, Phys. Rev. D 31, 2826 (1985).

24. R. Friedberg and T. D. Lee, Phys. Rev. D 16, 1096 (1977).

25. R. Friedberg and T. D. Lee, Phys. Rev. D 18, 2623 (1978).

26. W-Y. P. Hwang, Phys. Lett. B 116, 37 (1982).

27. W-Y. P. Hwang, Phys. Rev. D 29, 1465 (1984). 